\title{
¿El uso de las TIC reduce realmente la vulnerabilidad social en los jóvenes?
}

\author{
Silvia Lombarte Bel \\ slombarte@ub.edu \\ Universidad de Barcelona
}

\section{Introducción}

En este artículo se pretende hacer una aproximación a los posibles indicadores de vulnerabilidad de los jóvenes adolescentes de la sociedad actual relacionándolos con las TIC, para poder indagar sobre si el hecho de tener acceso y utilizar las TIC los hace menos vulnerables. En una primera parte del artículo situaremos el término vulnerabilidad social en el contexto de la sociedad actual, relacionándola con los cambios ocasionados por la introducción de las TIC.

Posteriormente pasaremos a hablar de las características de los jóvenes del siglo XX. A partir de aquí se citaran investigaciones que han indagado sobre indicadores de vulnerabilidad, para poder llegar a disminuirla, para que finalmente se pueda discutir si las TIC reducen o aumentan la vulnerabilidad de los jóvenes.

\section{1.¿Qué significa ser vulnerable en la sociedad actual?}

En la actualidad es sabido que nuestra sociedad está generando nuevos excluidos y sobretodo vulnerables, es decir, personas con posibilidad de exclusión social, debido a la inestabilidad y la liquidez instauradas en la sociedad (Bauman, 2002), tanto en las relaciones personales como en el sistema laboral. El término vulnerabilidad ha sido estudiado y definido por varios autores, pero sin duda uno de los más destacados es Castel (en Alvarez-Úria, 1992); este autor esquematiza que la zona de la vulnerabilidad social oscila ente la integración y la de exclusión. Según el autor encontraríamos tres franjas dentro de la sociedad: la de integración (caracterizada por un trabajo estable y una inscripción relacional sólida, tanto familiar como vecinal), la vulnerabilidad (zona inestable, caracterizada por un trabajo precario, paro intermitente y con frecuencia fragilidad del soporte familiar y social), por último la zona de marginalidad o exclusión (que se caracteriza por la carencia de trabajo y el aislamiento social). En la sociedad actual, encontramos autores como Cortarelo (1992) que defienden que la vulnerabilidad se ha extendido desde los márgenes de la sociedad hasta la centralidad, ampliando así la zona de vulnerabilidad social, y por tanto las posibilidades de ser excluido.

Otros autores, como Sennet (2000), han hablado de la realización de "fragmentos del trabajo", que estaría relacionado con el trabajo intermitente, debido a la instauración del capitalismo flexible. Como consecuencia de esta discontinuidad laboral, se produce una situación de desafiliación (Jolonch, 2001), que afecta a toda la población, aunque sin duda en algunos sectores de forma más notoria que en otros.

Como hemos podido constatar hasta aquí, el concepto de vulnerabilidad no es un fenómeno recién aparecido, pese que se está cambiando el contenido clásico de dicho término. Hasta ahora se había hablado de excluidos y vulnerables únicamente en términos estructurales[1], mientras en la actualidad 
se entiende el término en función de las condiciones de vida, aprendizaje y educación que rodean a las personas.

Para América Latina, Minujín ha considerado una noción que permite, por un lado, analizar la complejidad dinámica de las situaciones de pobreza y, por otro, identificar una zona de gradientes significativos entre los extremos: "inclusión/exclusión” $(1998,1999)[2]$.

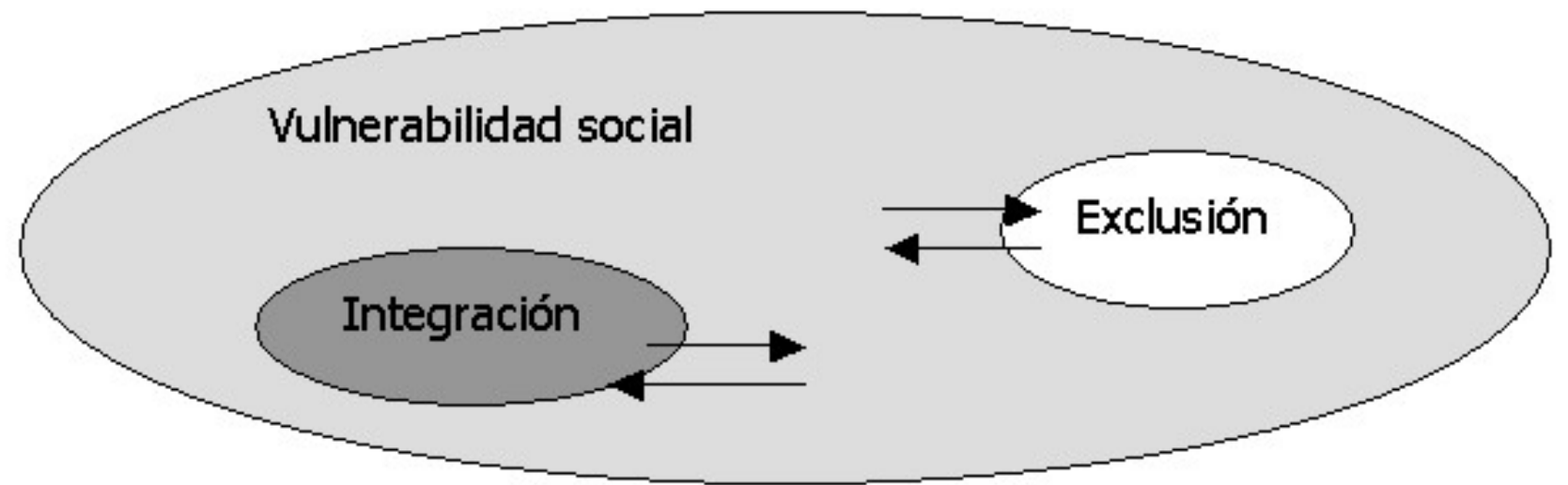

Así pues, en la sociedad actual, el fenómeno de la vulnerabilidad cobra cada vez más importancia, ya que el sistema estructural de la sociedad no solamente hace vulnerables a las clases bajas, sino también a las medias y altas.

Dentro de la vulnerabilidad encontraríamos una franja fronteriza con la exclusión social, que sería lo que llamaríamos "de riesgo social", definido como un grupo en proceso de desadaptación social por causas básicamente familiares (Balsells, 2003). Esta autora define el riesgo partiendo de tres enfoques: socio-ambientalista, relacionado con los factores contextuales, sus programas de intervención hacen hincapié en las familias; conductista, donde las situaciones de riesgo están causadas fundamentalmente por las dificultades de los padres en desarrollar su rol parental y en descubrir las necesidades básicas de los hijos; sistémico, que parte de la premisa de que el individuo con problemas forma parte de un sistema, básicamente familiar y con dificultades.

\section{Características de los jóvenes del siglo XXI}

Recordemos que la generación de los jóvenes de finales del siglo XX fue definida por la inestabilidad y constantes cambios (Beck, 1986). La generación @, se define como la generación en la quela mayoría de los jóvenes, aunque no de forma global, tienen acceso a Internet, lo cual tiene unas consecuencias directas en la vulnerabilidad del sujeto. Esta generación se encuentra dentro de la sociedad informacional (Castells, 1998), en la que la persona es más competente y más productiva cuanto más acceso y capacidad tiene de seleccionar información.

Feixa (2001) sostiene que si la última generación del siglo XX había sido bautizada como la generación $\mathrm{X}$, por el hecho de estar marcada por la incertidumbre y las paradojas de la crisis de las ideologías y el fin de la historia, la primera generación del siglo XXI, podría ser llamada "generación @”, para expresar las tendencias de cambio que están en juego en la actualidad tales como el acceso universal (aunque no general) a las nuevas tecnologías de la información y la comunicación[3]; la erosión de las fronteras tradicionales entro los sexos y los géneros, y el proceso de globalización cultural que conlleva a su vez nuevas formas de exclusión social.

Tal y como señalan Pereira y Marín (2001), sobre la importancia de aproximar la educación en la secundaria a los gustos de los jóvenes de hoy en día, "si uno de los principios básicos con más fuerza en la actualidad es la vinculación entre comunidad educativa y sociedad, la escuela no puede mantenerse ajena a esta realidad, es más, debe acortar distancias entre la vida de los escolares y el 
sistema educativo"[4]. En este artículo se introducía el trabajo educativo a través del cine; en la actualidad, como ya es sabido, la cultura de los jóvenes está basada en buena parte en el uso de las nuevas tecnologías, es por eso que al hablar de vulnerabilidad social se hace necesario no solamente contemplar las TIC, sino también, tenerlas en cuenta al analizar los factores que hacen al joven más vulnerable.

A pesar de la importancia que se le atribuye al uso de Internet, podemos constatar que no existen muchas investigaciones que relacionen el acceso a Internet y las consecuencias de éste. Según un estudio realizado en España por la consultora Media Planning[5] en febrero del 2004, un 50,3\% de los adolescentes entre 14 y 16 años utiliza Internet. En dicho estudio se concluye que las nuevas tecnologías, junto al cambio de la estructura familiar y las influencias multiculturales inciden en la manera en que el joven ve el mundo, se enfrenta e interactúa con él.

A parte de éstos, a Internet se le atribuyen otros peligros, como el acceso a la pornografía infantil, la pederastia, etc. En algunos países de la Unión Europea, como es el caso de Inglaterra, se a creado una red, llamada educaunet, con la pretensión de incrementar el uso seguro de Internet[6].

Pese a esto, debemos tener en cuenta que la capacidad de un joven para interpretar y hacer frente a este tipo de problemáticas, depende precisamente de sus competencias personales y del conocimiento que tenga de estos nuevos medios. Algunos investigadores han indagado en el llamado "uso patológico de Internet". Gracia Blanco (2002) relaciona las respuestas de personas que tienen disfunciones delante de la red (pérdida de control, pérdida de tiempo, acceso a páginas para adultos y chats) con las respuestas de los tests sobre ansiedad y disfunción social. Delante de estas situaciones se hace necesario que desde la educación se trabajen los aspectos de autorregulación delante del medio y la selección crítica de la información.

Para conocer con más profundidad las características de los jóvenes del siglo XXI, mencionaremos algunas investigaciones que han sido realizadas con jóvenes en relación a las TIC. En primer lugar, sintetizaremos el estudio "UK Children Go Online"'[7], realizado por el Departamento de Media y Comunicación de la London Schools of Economics and Political Science, publicado en Julio del 2004, en el cual se especifica que los temas más relevantes para crear marcos teóricos para análisis tanto académico como político en relación a las TIC (Livingston, 2004) son los siguientes:

* En relación al acceso, la desigualdad y la división digital; podemos concluir que en general el uso de Internet ha aumentado notoriamente en los últimos años. Pese a que el acceso en casa podría aumentar la división digital, la estrategia del gobierno de facilitar el acceso a través de las escuelas está ayudando ha disminuir las distancias entre las diferentes clases sociales. Así pues, mientras que el $75 \%$ de los jóvenes tienen acceso a Internet en casa, casi un 92\% lo tienen en la escuela.

* Otro aspecto que también se ha investigado es la naturaleza y la calidad de Internet; se ha comprobado que aunque no se dé de forma generalizada y pese a que los padres lo subestimen, se dan casos de acoso sexual (31\%), y en algunos casos comentarios desagradables a través del correo electrónico, chat o mensajería instantánea $(33 \%)$.

* Dentro de este mismo proyecto, también se resalta, la educación, el aprendizaje informal y la alfabetización digital; pese a que una de las prioridades políticas es la educación en las TIC, se ha comprobado que un $30 \%$ de los jóvenes no han recibido ningún aprendizaje sobre Internet.

*Por último, respecto a la comunicación, la identidad y la participación, pese a que las relaciones de amistad todavía se dan mayoritariamente cara a cara, se utiliza como primer modo de comunicación el móvil y como segundo el ordenador.

En otra investigación realizada por la Universidad de Murcia[8], sobre el uso de los medios de comunicación social por parte del alumnado de secundaria, se concluye que mientras la dotación de acceso a medios y equipamientos tecnológicos es más que aceptable, habiendo crecido enormemente 
la posesión individual de reproductores musicales y el móvil, los equipamientos tradicionales que implican un capital cultural para su uso y manejo, gozan de una presencia escasa (bibliotecas personales, lectura, etc). Como consecuencia de esta desigual distribución entre los medios de uno y otro tipo, segmentan a los hogares en dos tipos: unos dotados para el consumo de contenidos mediáticos dirigidos fundamentalmente al ocio, y otros que realmente utilizan, manejan y se sirven de la información.

\section{Aproximación a los indicadores de vulnerabilidad a través de las distintas investigaciones}

Los indicadores de vulnerabilidad de tipo estructural o macrosocial están relacionados con las condiciones de la sociedad, por las políticas, su historia y, por lo tanto, se hace difícil incidir de forma directa desde la educación; como consecuencia de estos factores, encontraríamos la inestabilidad en la que vivimos, la desigualdad social y la consecuente división digital.

Pese a esto existe otro tipo de características intrínsecas en la persona que llamaremos microsociales, en las que la educación tiene mucho que hacer, ya que dependerá de la situación y de la capacidad individual que la persona sea más o menos vulnerable.

a) Para definir algunos de los indicadores macrosociales, en primer lugar retomaremos a Castel, el cual especificaba la importancia de dos aspectos para que se diera la inclusión: estabilidad laboral y red de relaciones estables. En el caso de los adolescentes, podría traducirse de manera que el joven más vulnerable sería aquel que tuviera mayor fracaso escolar y una red de amistades más débil. De forma más concreta, podríamos catalogar como indicadores de vulnerabilidad macrosociales los investigados por el Instituto de Infancia y Mundo Urbano[9]. Por ejemplo, que el joven no tenga a su lado, por la razón que sea, las persona que ejerza su guarda; cuando presente pruebas de negligencia en su alimentación, higiene, salud o educación; cuando no haya los vínculos afectivos; cuando le falten los elementos básicos para su desarrollo integral.

Otro aspecto que también se ha tenido en cuenta es el índice de vulnerabilidad social generado a partir de los índices de vulnerabilidad ambiental, índice de población, y el mapa base de niveles de pobreza por poblaciones[10]. Estos indicadores de tipo macrosocial, que junto a los socioeconómicos (modelo de desarrollo) y político-institucional (capacidades y debilidades de los sistemas formales e informales), en los cuáles no pretendemos profundizar, dibujarían la situación social en la que el sujeto está inmerso y por lo tanto le afecta de manera directa.

b) Respecto a los indicadores microsociales, incluiremos factores de vulnerabilidad de tipo ideológico o cultural; en este aspecto encontraríamos la percepción de vulnerabilidad, debido a la experiencia acumulada y la historicidad de los procesos. Este aspecto que a priori nos puede parecer macrosocial, si lo analizamos de forma detenida podremos ver cómo la percepción que tenga el joven de si mismo podrá influenciar en que el sujeto sea un excluido o no; en este aspecto jugarían un papel importante los procesos de autopercepción y de autoestima, y es en estos en los que la pedagogía deberá tener un papel activo para que la persona tenga una percepción más inclusiva de si misma y de sus iguales. Pese que a priori no consideraremos el no acceso a las TIC como factor de inclusión y por lo tanto de vulnerabilidad, sí que podemos constatar que tiene cierta relación a la hora de desarrollar algunas capacidades. Así pues, siguiendo las investigaciones de Hellawell (2001) podemos ver cómo la utilización de las nuevas tecnologías puede ayudar a desarrollar nuevas capacidades y a mejorar la empleabilidad y la confianza en uno mismo. Así mismo en relación a las capacidades cuyo desarrollo nos hacen menos vulnerables, citando a Martínez y Buxarrais (en prensa), destacaríamos los siguientes: aprender a aprender de forma autónoma; adaptarse a los cambios; construir conocimiento a partir de la información proveniente de las TIC; demostrar una actitud pro-activa y emprendedora; interés y compromiso en los proyectos colectivos; estar entrenado en la construcción de la propia identidad; ser capaz de tomar decisiones que afecten el propio interés, pero también el de los otros, etc. Además de estos indicadores, encontraríamos lo que Brader (2001) también señala el aspecto subjetivo de vulnerabilidad social, muy inestable y ligado a las acciones de la persona. Citando los estudios de Lohman y Reitzes (1954), Brader mantiene que, al preguntar a una persona si se siente 
excluida o no (por lo tanto indagará sobre si es más o menos vulnerable), su respuesta variará según la situación en que se encuentre en ese momento ( si está sola, acompañada o conectada a Internet). Es decir, dirá que se siente más o menos excluida en función de su situación actual; por lo tanto, su autodefinición y su autoestima variarán. Este mismo autor narra su experiencia como educador en un centro de ocio para jóvenes en el que se utilizan las nuevas tecnologías. Brader explica que el trabajo educativo en este centro no era rígido y formal, sino flexible y adaptable. Este tipo de metodología proporcionaba a los jóvenes oportunidades para elegir en función de sus intereses. Así pues, podemos decir que la educación en contextos no formales, en los que se promueva la utilización de las TIC para realizar actividades imaginativas y artísticas, puede ser un camino para motivar a los grupos sociales más vulnerables.

\section{Discusión}

Pese a que no hay duda de que hay ciertos aspectos estructurales de la sociedad que desde el origen hacen más vulnerables a unas personas que a otras, después de profundizar sobre dicho concepto, sobre el estado de la juventud de hoy en día y hacer un recorrido por diferentes investigaciones sobre el tema, podemos ver cómo la educación tiene mucho que hacer en la disminución de este fenómeno.

De forma indudable apoyaremos la tesis de Castel, la cual nos relaciona la vulnerabilidad con la inestabilidad laboral y la fragilidad familiar y social. La sociedad actual se caracteriza por esta inestabilidad; por lo tanto lo que deberá procurar la educación es la capacidad de insertarse laboralmente, o como mencionaba Brader, la mejora de la empleabilidad (y aquí entrarían muchas capacidades tales como: la autopercepción, la autoestima, el desarrollo de nuevas capacidades, la confianza en uno mismo, aprender a aprender de forma autónoma, adaptarse a los cambios, etc), y por otro lado la capacidad de mantener una redes de relaciones (sociales y personales) y en caso de que carezcan, la creación de éstas.

Otras de estas capacidades que también han sido mencionadas tales como demostrar una actitud proactiva y emprendedora, interés y compromiso en los proyectos colectivos o ser capaz de tomar decisiones que afecten el propio interés, pero también el del grupo, pueden afectar tanto a la empleabilidad como a la consolidación de la red de relaciones.

La paradoja final es que para lograr el acceso a las TIC, las personas deben estar "alfabetizadas digitalmente", que se define como el conjunto de hábitos a través de los cuales se usan las nuevas tecnologías de la información y la comunicación para aprender, trabajar, socializar, divertirse, etc. (Ba, Tally \& Tsicalas, 2002). Por un lado, podemos apoyar la hipótesis de que todas estas capacidades, habilidades y hábitos desarrollados a través de las nuevas tecnologías pueden ser extrapolables a otros ámbitos de la vida social y profesional, pero por otro lado no podemos afirmar que el hecho de tener más acceso a las TIC nos haga automáticamente menos vulnerables, ya que en primer lugar hace falta estar alfabetizado digitalmente y por otro se deben desarrollar una serie de capacidades para poder hacer frente a las TIC, además de estar imbricado en una red de apoyo familiar o social sostenible.

En resumen, la brecha digital se agranda cuando menos acceso hay ante las TIC por parte de los jóvenes. Pero ¿qué pasa cuando el uso se da de forma incontrolada y/o sin planificación? Probablemente, se puede llegar a ser más vulnerable, y aquí entran en juego el dominio de una serie de capacidades[11]:

Autoconocimiento y autoestima. Es importante la clarificación de nuestra forma de ser, pensar y sentir; si somos capaces de definirnos a nosotros mismos y a descubrir a que le otorgamos sentido a nuestra vida, podremos hacer uso de las TIC con unos objetivos concretos y una orientación definida, lo cual nos permitirá optimizar el tiempo de uso de estas con la pretensión de alcanzar los objetivos propuestos de forma efectiva.

Autonomía y autorregulación. La autonomía es la capacidad que permite determinarse a uno mismo, mientras que la autorregulación se hace necesaria para poder promover la autonomía de voluntad y 
una mayor coherencia de la acción personal. Así pues, las personas que tengan desarrollada esta competencia, serán más capaces de utilizar las TIC de forma controlada (tanto el tiempo de conexión como el tipo de actividad: búsqueda de información y/o recursos, juegos, correo electrónico, mensajería instantánea, etc), y a su vez, saber encontrar la información y gestionarla.

Capacidad de diálogo. Esta capacidad supone poder intercambiar opiniones y razonar sobre los diferentes puntos de vista e intentar llegar a un consenso, a un acuerdo justo racionalmente motivado. El desarrollo previo de esta capacidad antes del uso de las TIC nos permitirá que delante de un sistema de comunicación instantánea, como son las TIC, seamos capaces de reflexionar motivadamente a la hora de establecer un diálogo. El uso de las TIC supone muchas veces el no escuchar al interlocutor, es por eso que se debe estar preparado para dialogar a través de las TIC, sobre todo en esta sociedad dónde muchos de los estudios y trabajos se realizan a través de la red.

Empatía y perspectiva social. Las TIC abren la ventana a un mundo de realidades muy diversas que permiten el conocimiento y acercamiento a personas muy diversas. Es importante que seamos capaces de estar abiertos a aprender de lo nuevo que nos aportan, pero también a poder discernir lo que no nos favorecerá para lograr nuestro proyecto de vida.

Capacidad para transformar el entorno. Esta capacidad nos permite implicarnos en proyectos colectivos y buscar bienes comunes que supongan la superación. Las TIC pueden favorecer la implicación en proyectos colectivos, pero antes de esto hace falta haber desarrollado la capacidad de descentrar nuestros propios intereses y buscar objetivos para mejorar la situación de diferentes colectivos.

Habilidades sociales y para la convivencia. Estas son las capacidades que permiten la coherencia entre los criterios personales, las normas y los principios sociales. A su vez estas capacidades facilitan la interrelación y la comunicación positiva con los demás. Por eso es importante tener claros dichos criterios a la hora de comunicarnos, ya sea de forma expositiva o interactiva con el entorno.

Desarrollo del razonamiento moral. Tiene como finalidad llegar a pensar según criterios de justicia y dignidad personal, teniendo en cuenta los principios de valor universales. A través de las TIC podemos comunicarnos con personas que tienen puntos de vista muy diferentes, por lo tanto hemos de ser capaces de respetar las otras opiniones y estar preparados también para los posibles conflictos cognitivos, creando interrogantes en nuestras estructuras de pensamiento y planteando de nuevo nuestras opiniones.

Comprensión crítica. La definiremos como la capacidad de adquirir un conocimiento y el análisis critico de la realidad contextualizando y contrastando los diversos puntos de vista, mediante una actitud de entendimiento y compromiso con la pretensión de mejorarla. Esta capacidad tiene especial importancia, ya que delante de la cantidad de estímulos que recibimos, debemos estar preparados para prestar especial atención a aquellos que realmente nos interesen, y por otro lado ser capaces de comparar, de buscar diferentes versiones y de no creer de forma inminente todo aquello que las TIC transmiten.

A modo de conclusión pues, podemos decir que el uso de las TIC no provoca de forma inmediata la reducción de la vulnerabilidad social, sino que el sujeto necesita tener una serie de capacidades para poder enfrentarse a estas de forma efectiva y controlada. En este sentido hace falta investigar más, no solamente en términos de acceso o no acceso, sino que también de cómo se da el uso creativo y reflexivo de las TIC. Pese a que algunos de los estudios anteriores mencionaban la necesidad de desarrollar una serie de capacidades, ninguno de estos ha descrito dichas capacidades y tampoco han sido ni investigadas, ni estudiadas con profundidad. Así pues, al relacionar la vulnerabilidad social con las TIC, no podemos afirmar que aquellos que utilizan las TIC sean menos vulnerables, ya que en algunos casos el hecho de usar las TIC sin el desarrollo de unas capacidades previas podría producir el efecto contrario, es decir, el acceso de la vulnerabilidad. 


\section{Bibliografía}

Ba, H; Tally, W; Tsikalas, K. (2002). Investigating Children's Emerging Digital Literacies. Caroline, A \& Peters, S Lynch School of Education: Boston College (www.jtla.org)

Balsells, M. A. (2003) La infancia en riesgo social desde la sociedad del bienestar, Revista electrónica Teoria de la Educación: educación y cultura en la sociedad de la información, [http://www3.usal.es/ teoriaeducacion/rev_numero_04/n4_art_balsells.htm]

Ballesta, J (dir) (2003) El consumo de los medios en los jóvenes de secundaria. Madrid: Editorial CCS Bauman, Z (2002) Modernidad líquida. Argentina: Fondo de cultura económica

Brader, A. (2001). Young people's use of ITC's: a practitioners perspective. Bolton: Presented at the Ethics, ITC and Social Exclusion Conference

Buxarrais, M.R; Martinez, M. (en prensa) Children's rights in the society of information and technologies, Canada: Encounters on Education.

Castel, R. (1992). "La inserción y los nuevos retos de las interviones sociales" a ÁLVAREZ-ÚRIA, F(COMP.). Marginación e inserción. Los nuevos retos de las políticas sociales. Madrid: Endymión.

Castells, M. (1998) La era de la información. Economía, sociedad y cultura. Vols I-II-III. Madrid: Alianza.

Gracia Blanco, M; Vigo Anglada; Fernández Pérez, Mª José; Marcó Arbones; Maria (2002), "Problemas conductuales relacionados con el uso de internet: un estudio exploratorio", Anales de psicología, vol. $18 \mathrm{n}^{\circ}$ 2, diciembre 2002.pp. 273-298

Feixa, C. (2001) Generació @. La joventut al segle XXI, Barcelona: Secretaria General de Joventut.

Ferrés, J. (1994) Televisión y educación. Barcelona: Paidós.

Hellawell, S. (2001). Beyond Access, ICT and social inclusion. Fabian society: London

Jollonch, A. (2002) Educació i infància en risc. Barcelona: CETC. Pòrtic.

Livingstone, S (2004): Regulation internet at home: contrasting the perspectives of children and parents.MEDIA@LSE: Londres. Accesible on-line a: http://www.children-go-online.net.

Sennet, R. (2002) La corrosión del carácter. Madrid: Anagrama.

\section{Notas:}

[1] Nos referimos a estructural cuando los términos hacen referencia a propiedad de las sociedades, es decir, aquellos factores que dependen de la estructura social y de la historia más que de las personas.

[2] www.aiaccproject.org/meetings/ SanJose_03/Session4/Session4_CNatenzonB.doc [15/11/2004]

[3] Con respecto al acceso universal a las nuevas tecnologías se da una posición optimista y otra más bien pesimista: mientras algunos opinan que la tecnología revolucionará las dinámicas mundiales actuales, otros la interpretan como una invasión cultural, o peor, como un peligro tangible para las personas más vulnerables. Esta visión más pesimista de los medios es la que reclama a la educación que actúe dando las estrategias necesarias para poder hacer frente al impacto de los medios. También cabe destacar la importancia del ámbito de la comunicación desde dos tipos de entornos, la 
información y la comunicación, ya que están proporcionando modelos, estereotipos y ideologías, y en consecuencia están también contribuyendo en la configuración de la personalidad.

[4] $\mathrm{M}^{\mathrm{a}}$ Carmen Pereira Domínguez y $\mathrm{M}^{\mathrm{a}}$ Victoria Marín Valle, Respuestas docentes sobre el cine como propuesta pedagógica. Análisis de la situación en Educación Secundaria. Teoría de la educación, Vol. 13. 2001, pp. 235

[5] Este estudio puede ser consultado en la web [www.marketingdirecto.com/estudios/estudio.php]

[6] Consultar a www.educaunet.org

[7] Puede consultarse a: www.children-go-online.net

[8] Ballesta, J (dir) (2003): El consumo de los medios en los jóvenes de secundaria. Editorial CCS, Madrid.

[9] Observatorio sobre infancia y juventud que depende del Ayuntamiento de Barcelona. [www.ciimu.org]

[10] Para más información sobre el cálculo de los índices puede consultase la página web del Centro Internacional de Agricultura Tropical (http://gisweb.ciat.cgiar.org/Vulnerabilidad/social.htm)

[11] Estas capacidades han sido descritas y evaluadas por el GREM (Grupo de Investigación de Educación en Valores, de la Universidad de Barcelona). Cita: Noguera, E.; Tey, A.; Buxarrais, M.R.; Martínez, M.; Prats, E.; "Estrategias de educación en valores" La educación en valores en Iberoamérica. Foro Iberoamericano sobre Educación en Valores. Madrid: Organización de Estados Iberoamericanos para la Educación, la Ciencia y la Cultura, 2001, pp. 53-89.

(C) Ediciones Universidad de Salamanca 